\title{
TORSION FREE SUBGROUPS OF FUCHSIAN GROUPS AND TESSELLATIONS OF SURFACES ${ }^{1}$
}

\author{
BY ALLAN L. EDMONDS, JOHN H. EWING AND RAVI S. KULKARNI ${ }^{2}$
}

It has been known for many years that a finitely generated fuchsian group $G$ i.e. a finitely generated discrete subgroup of orientation-preserving isometries of the hyperbolic plane contains a torsion free subgroup of finite index. The known proofs are by representations in the symmetric groups cf. Fox [3] or by the method of congruence subgroups cf. Mennicke [4]. The latter method extends to all finitely generated matrix groups cf. Selberg [5]. There is no information about the possible indices of subgroups in these proofs. Here we announce the precise determination of the possible indices of torsion free subgroups of finite index in terms of the torsion in $G$. Using the connection between fuchsian groups and uniformization of Riemann surfaces the results may be interpreted as a step in determining a class of intermediate uniformizations, or looked in a different way, a step towards a topological classification of holomorphic maps between Riemann surfaces of finite type. Contained herein are some results of a naive geometric interest. Namely they imply the existence of certain interesting tessellations of surfaces which are natural generalizations of the tessellations of the sphere determined by Platonic solids. We remark that we completely leave aside the questions of normality of subgroups. Determining the indices of normal torsion-free subgroups of finite index in fuchsian groups appears to involve deeper number theoretic considerations which are probably yet to be understood.

To formulate our main result let $G$ have a standard presentation with generators $a_{1}, b_{1}, \ldots, a_{g}, b_{g}, x_{1}, \ldots, x_{r}, y_{1}, \ldots, y_{s}$ and relations $x_{1}^{m_{1}}=\cdots=$ $x_{r}^{m_{r}}=1$ and $a_{1} b_{1} a_{1}^{-1} b_{1}^{-1} \cdots a_{g} b_{g} a_{g}^{-1} b_{g}^{-1} x_{1} \cdots x_{r} y_{1} \cdots y_{s}=1$. Let $l=$ $\operatorname{LCM}\left\{m_{1}, \ldots, m_{r}\right\}$, and let $l_{(2)}$ denote the 2-primary part of $l$. We say that $G$ has odd type if $s=0, l_{(2)}>1$, and the number of $m_{i}$ 's such that $l_{(2)} \mid m_{i}$ is odd. Otherwise $G$ has even type.

THEOREM 1. The infinite fuchsian group $G$ contains a torsion free subgroup of index $k$ if and only if $k \equiv 0$ modulo $2^{\epsilon} l$, where $\epsilon=0$ if $G$ has even type and $\epsilon=1$ if $G$ has odd type.

Received by the editors April 30, 1981. $05 \mathrm{~B} 45$.

1980 Mathematics Subject Classification. Primary 30F35; Secondary 57M12, 51 M20,

Key words and phrases. Fuchsian groups, tessellations, branched coverings, surfaces.

${ }^{1}$ Supported in part by grants from the National Science Foundation.

${ }^{2}$ Guggenheim Fellow. 
An alternate formulation is the following. A torsion free subgroup of finite index in a finitely generated fuchsian group is isomorphic to $\pi_{1}(M)$ where $M$ is an oriented surface of finite type. According to the theorem, conversely, given $H \approx \pi_{1}(M), M$ an oriented surface of finite type and $G=$ a finitely generated fuchsian group $H$ embeds in $G$ as a subgroup of index $k<\infty$ iff the RiemannHurwitz condition is fulfilled i.e. more succinctly $\chi(H) / \chi(G)=k$ where $\chi$ denotes the Euler characteristic of a group in the sense of Wall.

The proof of this theorem is given in detail in [2]. In case $G$ has positive genus $(g>0)$ or $G$ is not cocompact $(s>0)$, the result is easy. For one can produce a homomorphism to an appropriate dihedral or cyclic group and realize the asserted subgroup as the inverse image of a subgroup of order 2 or 1 . The remaining case of Dyck groups $(g=0$ and $s=0)$ is much more subtle. For example the $(2,3,7)$ triangle groups admits no nontrivial homomorphism to a finite solvable group.

Our approach to the theorem is to observe that the existence of a torsion free subgroup of $G$ is equivalent to a certain tessellation on a surface of finite type. Precisely to take care of the Dyck groups we prove

TheOREM 2. Let $M$ be a closed, orientable surface and let $m_{1}, m_{2}, \ldots$, $m_{r}, R, E, V_{1}, \ldots, V_{r}$ be positive integers satisfying

$$
2 E=r R ; \quad 2 m_{i} V_{i}=R, i=1, \ldots, r ; \quad \text { and } \quad R-E+\sum_{i=1}^{r} V_{i}=\chi(M) .
$$

Then there is a tessellation of $M$ into $R$ r-gons with $E$ edges and $\Sigma_{i=1}^{r} V_{i}$ vertices, $V_{i}$ of valence $2 M_{i}(i=1, \ldots, r)$ such that each region has vertices of valence $2 M_{1}, \ldots, 2 M_{r}$ up to cyclic order.

The proof contains an involved sequence of explicit constructions of tessellations coupled with inductions and branched covering arguments.

The case of $(p, q, 2)$ triangle groups has especially nice geometric import. In this situation the regions of the tessellation corresponding to a torsion free subgroup of index $k$ can be combined in groups of $2 p$ to form a tessellation by $k / 2 p p$-gons in which each vertex has valence $q$. Conversely, such a tessellation, generalizing the Platonic solids, corresponds to a subgroup of the $(p, q, 2)$ triangle group. We have dealt with this special case of the general problem, including here the more difficult nonorientable analogue, in [1].

Added In Proof. (1) The first and the third authors, in collaboration with R. E. Stong have further obtained various results concerning inclusions of one finitely generated fuchsian group into another as a subgroup of finite index. Besides the obvious Riemann-Hurwitz condition and the "genus and cusp" conditions there appears a new diophantine condition which is necessary but not in 
general sufficient for the existence of an associated branched covering of surfaces. The existence of branched coverings is established by algebraic or tessellation methods. The cases (1) genus $>0$ or (2) the number of punctures $>0$ or (3) the number of elliptic branch points $>3$ are relatively easy to handle. The remaining case - precisely that corresponding to subgroups of the Schwarz triangle groups - is investigated by relating it to bicolorable triangulations of closed surfaces.

(2) The third author has further investigated the indices of normal subgroups in finitely generated fuchsian groups. The following typical result illustrates the connections of this problem to finite simple groups and distribution of primes in arithmetic progressions. Fix primes $p<q$. Let $\theta_{p, q}(x)$ be the number of primes $r \leqslant x$ such that the minimum index of a normal subgroup in the $\{p, q, r\}$-triangle group with quotient $\approx$ a known finite simple group $\not \operatorname{PSL}_{2}\left(\mathbf{F}_{s}\right)$ where $\mathbf{F}_{s}$ denotes a finite field with $s$ elements. Let $\pi(x)$ be the number of primes $\leqslant x$.

THEOREM. If the extended Riemann hypothesis is valid then

$$
\operatorname{Lim}_{x \rightarrow \infty} \theta_{p, q}(x) / \pi(x)=0 .
$$

Roughly speaking the theorem says that a quotient of minimum order of the $\{p, q, r\}$-triangle group with torsion free kernel is "almost always" $\approx$ $\mathrm{PSL}_{2}\left(\mathbf{F}_{s}\right)$ for some $s$ - the exceptions do occur, but they have "zero density".

\section{REFERENCES}

1. A. L. Edmonds, J. H. Ewing, and R. S. Kulkarni, Regular tessellations of surfaces and $(p, q, 2)$ triangle groups, Ann. of Math. (to appear).

2. - Torsion free subgroups of Fuchsian groups and tessellations of surfaces (to appear).

3. R. Fox, On Fenchel's conjecture about F-groups, Mat. Tidsskr. B (1952), 61-65.

4. J. Mennicke, Eine Bemerbung über Fuchssche Gruppen, Invent. Math. 2 (1967), 301-305; Corrigendum, ibid 6 (1968), 106.

5. A. Seiberg, On discontinuous groups in higher-dimensional symmetric spaces, Contributions to Function Theory, Tata Institute of Fundamental Research, Bombay, 1960, pp. $161-183$.

DEPARTMENT OF MATHEMATICS, SWAIN HALL EAST, INDIANA UNIVERSITY, BLOOMINGTON, INDIANA 47405 